\title{
FOXM1 as chemo-sensitizing target in neuroendocrine lung tumors.
}

\author{
F. Christen ${ }^{1,2}$, I. Grass ${ }^{1,3}$, L. Worpenberg ${ }^{1,3}$, F. Lewens ${ }^{1}$, F. Briest ${ }^{1,3,4}$, P. Grabowski ${ }^{1,4}$
}

${ }^{1}$ Dept. of Gastroenterology, Infectious Diseases, Rheumatology, Charité - Universitätsmedizin Berlin, Campus Benjamin Franklin, Germany; ${ }^{2}$ Institute of Biology, Humboldt-Universität Berlin, Germany ${ }^{3}$ Dept. of Chemistry and Biochemistry, Freie Universität (FU) Berlin, Germany; ${ }^{4}$ Dept. of Gastroenterology and Endocrinology, Zentralklinik Bad Berka GmbH, Germany,

Background:

Bronchopulmonary neuroendocrine neoplasms (BP-NEN) represent a heterogeneous subgroup among lung tumors Due to their clinical and molecular characteristics BP-NEN are grouped into low-grade typical carcinoids (TC) intermediate-grade atypical carcinoids (AC), and high-grade large-cell neuroendocrine lung carcinomas (LCNEC) and small cell lung carcinomas (SCLC). For the highly malignant carcinomas combined chemotherapy is the standard therapy option, but shows its limitations in recurring tumors [1]. For advanced carcinoid tumors somatostatin-analogues are firstline treatment, since they are often insensitive to chemotherapy [2]

FOXM1 is a typical proliferation-associated transcription factor and is involved in cell cycle control and DNA damage response. FOXM1 has been implicated in tumor progression and is therefore a promising target in many cancer types [3] Additionally, inhibition of FOXM1 has been shown to enhance sensitivity to chemotherapy [4]

Aim:

To investigate the role of FOXM1 in BP-NEN, it was analyzed whether inhibition of this transcription factor, eithe by RNA interference or proteasome inhibition, has a chemo sensitizing effect. Proteasome inhibitors have been shown to target FOXM1 either directly or indirectly [5]. Here, the clinically relevant proteasome inhibitor bortezomib was used, since it has been proven promising in SCLC [6]

Material and Methods:

BP-NEN cell lines (NCl-H727=TC, NCl-H810=LCNEC, NCl$\mathrm{H} 69=\mathrm{SCLC}$ ) and the non-neuroendocrine NSCLC cell line A549 were transfected with siRNA against FOXM1 or control siRNA and subsequently treated with cisplatin. Additionally, the same cell lines were treated with bortezomib, cisplatin or a combination of both, and analyzed via cell proliferation assay (WST-1 cell proliferation assay, Roche). Molecular effects of FOXM1 knockdown as well as the inhibitors bortezomib and cisplatin were investigated by flow cytometry (cell cycle: propidium iodide staining for DNA content and phospho-Histone $\mathrm{H} 3$ for mitotic index; apoptosis: JC-1 assay for detection of mitochondrial membrane polarization), western blot and multiplex gene expression assay (nCounter ${ }^{\circledR}$ PanCancer pathway panel, Nanostring technologies, 770 genes).

Results:

Bortezomib is highly effective in BP-NEN cell lines and exerts synergistic effects with cisplatin (figure 1). It induces G2 arrest, and in combination with cisplatin a slight shift to apoptosis is seen in $\mathrm{NCl}-\mathrm{H} 727$ and $\mathrm{NCl}-\mathrm{H} 810$ (figure 2) $\mathrm{NCl}-\mathrm{H} 69$ cells show no induction of apoptosis after knockdown of FOXM1, whereas the NSCLC (wild-type p53) cell line is highly apoptotic (figure 3 ). The DNA damaging agent cisplatin leads to increased DNA repair mechanisms, which are decreased after inhibition of FOXM1 (figures 4 and 5)

\section{Conclusion:}

Inhibition of FOXM1 sensitizes BP-NEN cell lines to apoptosis induced by DNA damaging agents. The described effects are much stronger in the p53 wild-type cell line A549, whereas BP-NEN are often p53 mutated. It might be beneficial to improve the mode of application, since cisplatin exerts its main effects in $\mathrm{G} 1$ and $\mathrm{S}$ phase, which might be counteracted by the increased $\mathrm{G} 2$ arrest after inhibition of FOXM1. An application route with induction of DNA damage and $S$ phase arrest before DNA repair is impaired by inhibition of FOXM1, might increase the apoptotic response. Nevertheless, inhibition of FOXM1 is a promising approach to re-sensitize BP-NEN to chemotherapy with cisplatin, which is especially relevant in high-grade BP carcinomas. In this context, bortezomib seems a promising clinically relevant agent to facilitate FOXM1 inhibition.

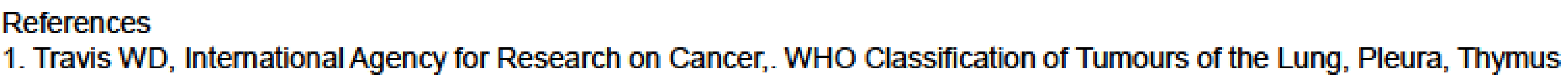

R. Trevisus WD, Intemational Agency for Research on Cancer. WHO Classification of Tumours of the Lung, Pleura, ThymIS

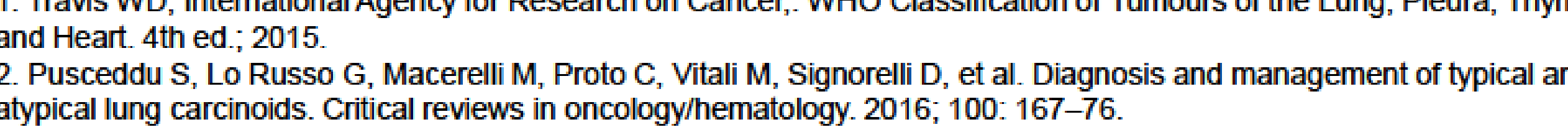

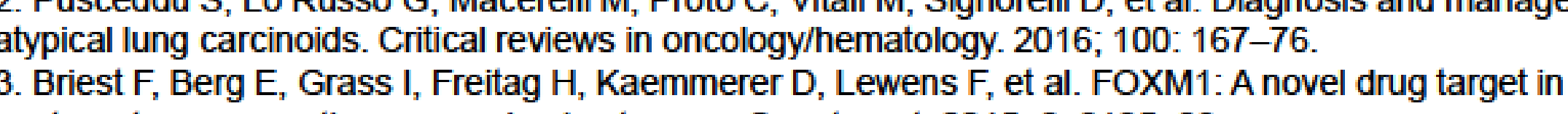

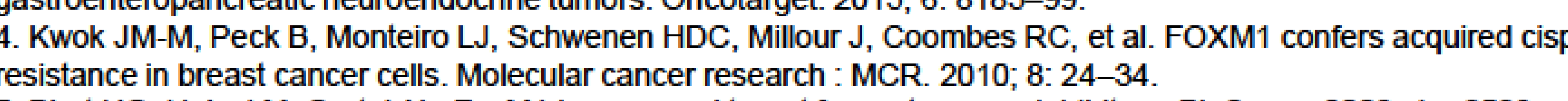

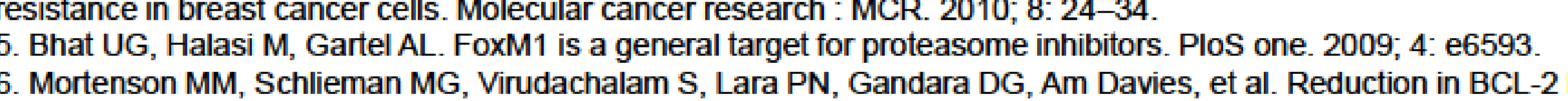

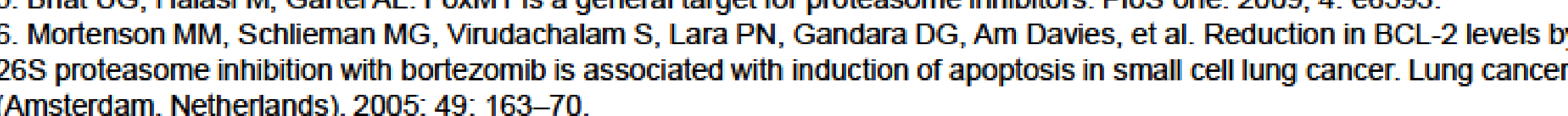
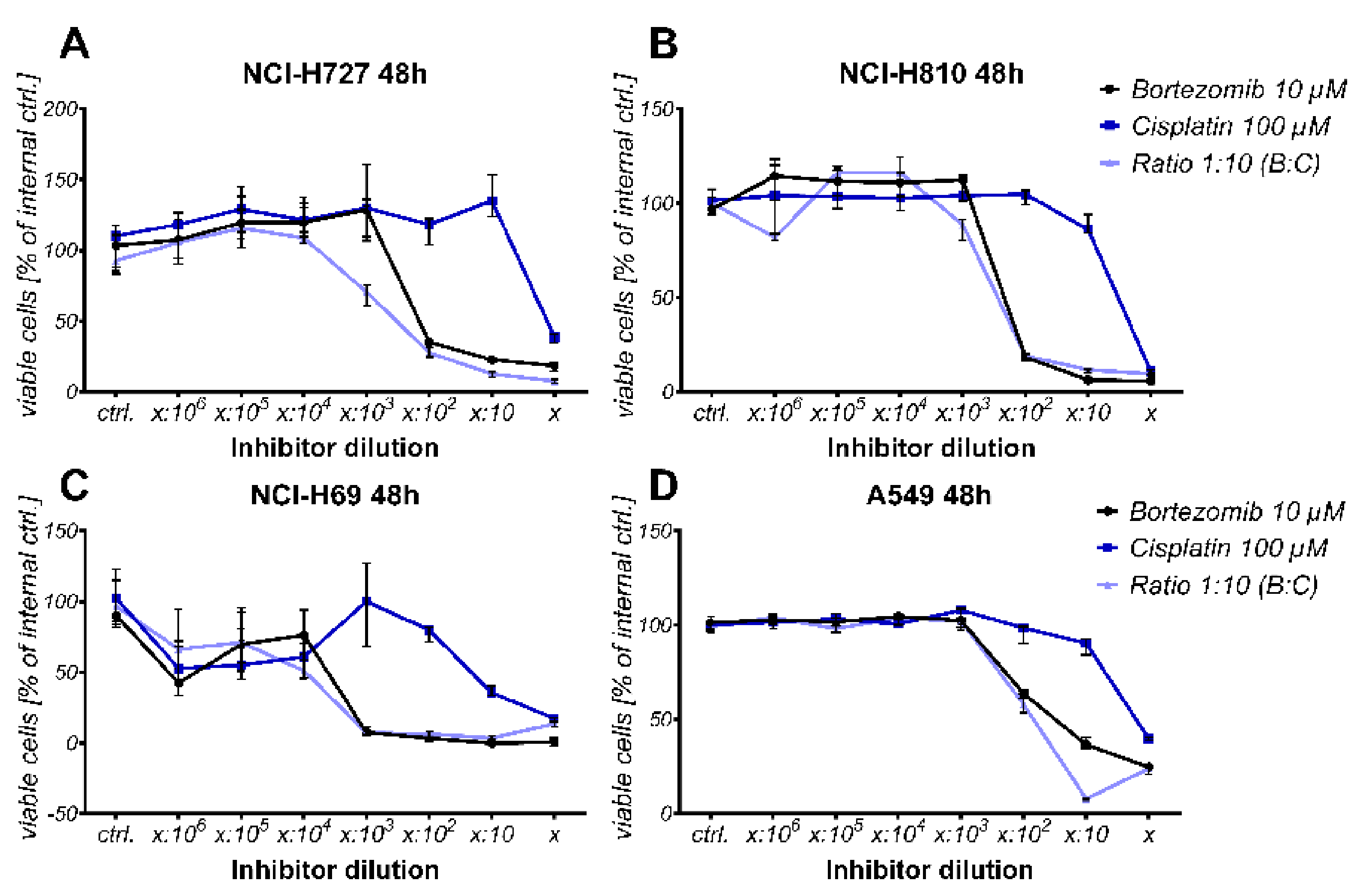

Figure 1: WST-1 cell proliferation assay after bortezomib and cisplatin treatment, as well as a combination of both substances. Cells were incubated with the single substances and a combination of both in a 1:10 ratio for $48 \mathrm{~h}$. A NClH727 showed strongly enhanced anti-proliferative effects after combination treatment compared to the related mono therapies (IC50 $\mathrm{IC} \mathrm{b}_{\text {bortez }} \sim 80 \mathrm{nM}$ ). $\mathrm{B}+\mathrm{C} \mathrm{NCl}-\mathrm{H} 810$ and $\mathrm{NCl}-\mathrm{H} 69$ showed also enhanced anti-proliferative effects after combination treatment. (IC50 $\sim 25 \mathrm{nM}$ vs. IC $50_{\text {b }} \sim 50 \mathrm{nM}$, and IC $50_{\text {comb }} \sim 25 \mathrm{nM}$ vs. 1 C50 bortez $\sim 10$ nM, respectively) (D) A549 show on bonez 10 in proliferative effect after substance combination.

A: Inhibition of FOXM1 by bortezomib

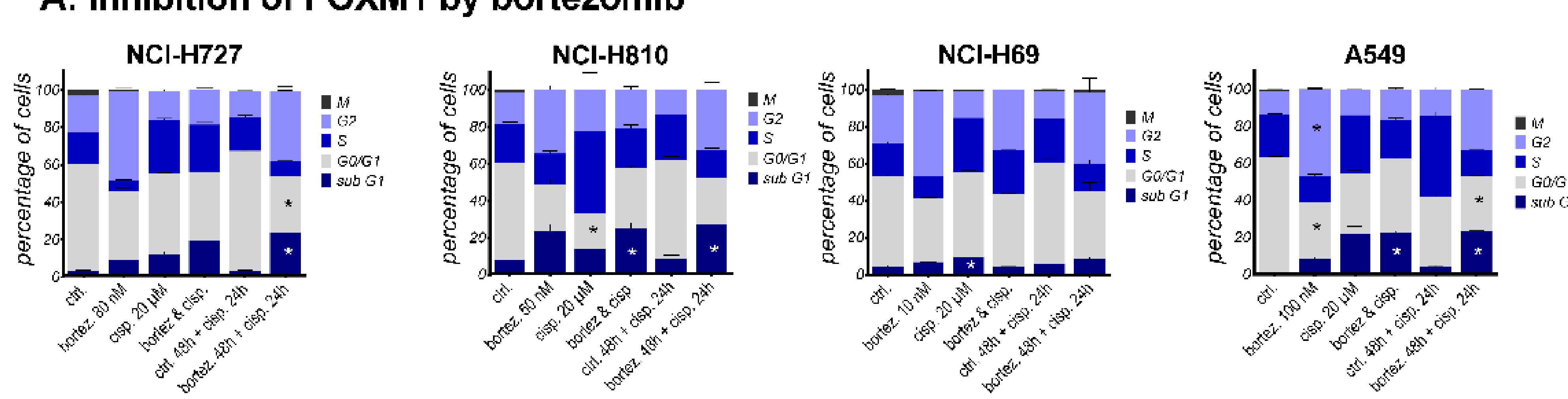

Figure 2: FACS analysis of the cell cycle and mitotic index.

A Cells were treated with bortezomib cisplatin (48h) and combinations of both inhibitors applied simultaneously or sequentially. Bortezomib increases sub $\mathrm{G} 1$ and $\mathrm{G} 2$ cell population in al cell lines. Cisplatin leads to an increase in $S$ phase cells. Combination therapy increases apoptosis (sub G1) and either $\mathrm{G} 2$ arrest or $S$ phase arrest depending on the mode of application.

B: Inhibition of FOXM1 by RNA interference

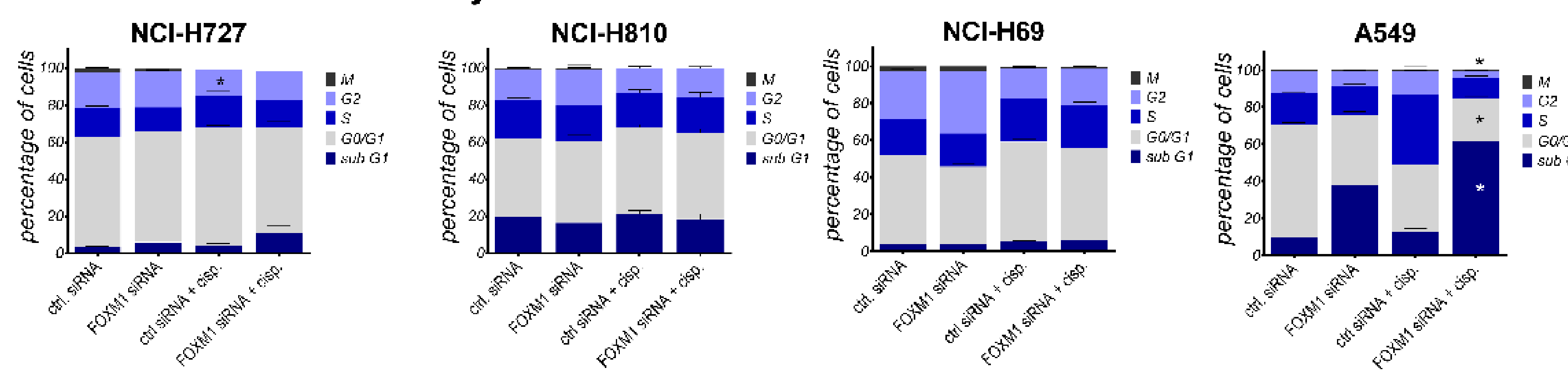

B Cells were transfected with siRNA targeting FOXM1 or control siRNA versus combination with cisplatin. In $\mathrm{NCl}-\mathrm{H} 69$ a G2 arrest is noticeable, whereas in the p53 wildtype cell line enhanced after is induced and greatly

A

B

C

D
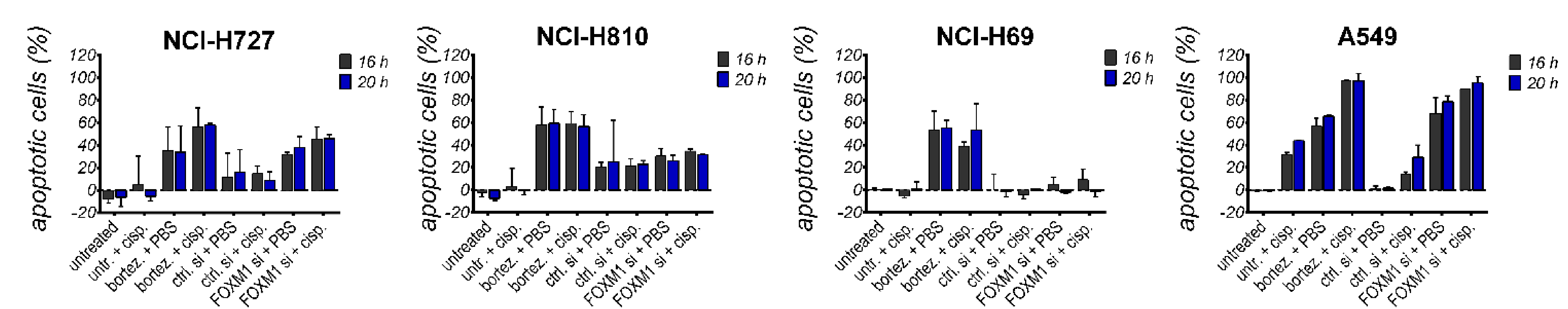

Figure 3: Detection of early apoptosis by JC-1 FACS analysis after $16 \mathrm{~h}$ and $20 \mathrm{~h}$. A+B In $\mathrm{NCl}-\mathrm{H} 727$ and $\mathrm{NCl}-\mathrm{H} 810$ apoptosis is induced after inhibition of FOXM1 by bortezomib and siRNA, which is enhanced after combination with cisplatin. C NCI-H69 cells show induction of apoptosis after bortezomib treatment, which is not enhanced after combination. D A549 show strong induction of apoptosis after FOXM1 inhibition, reaching almost $100 \%$ after combination with cisplatin.

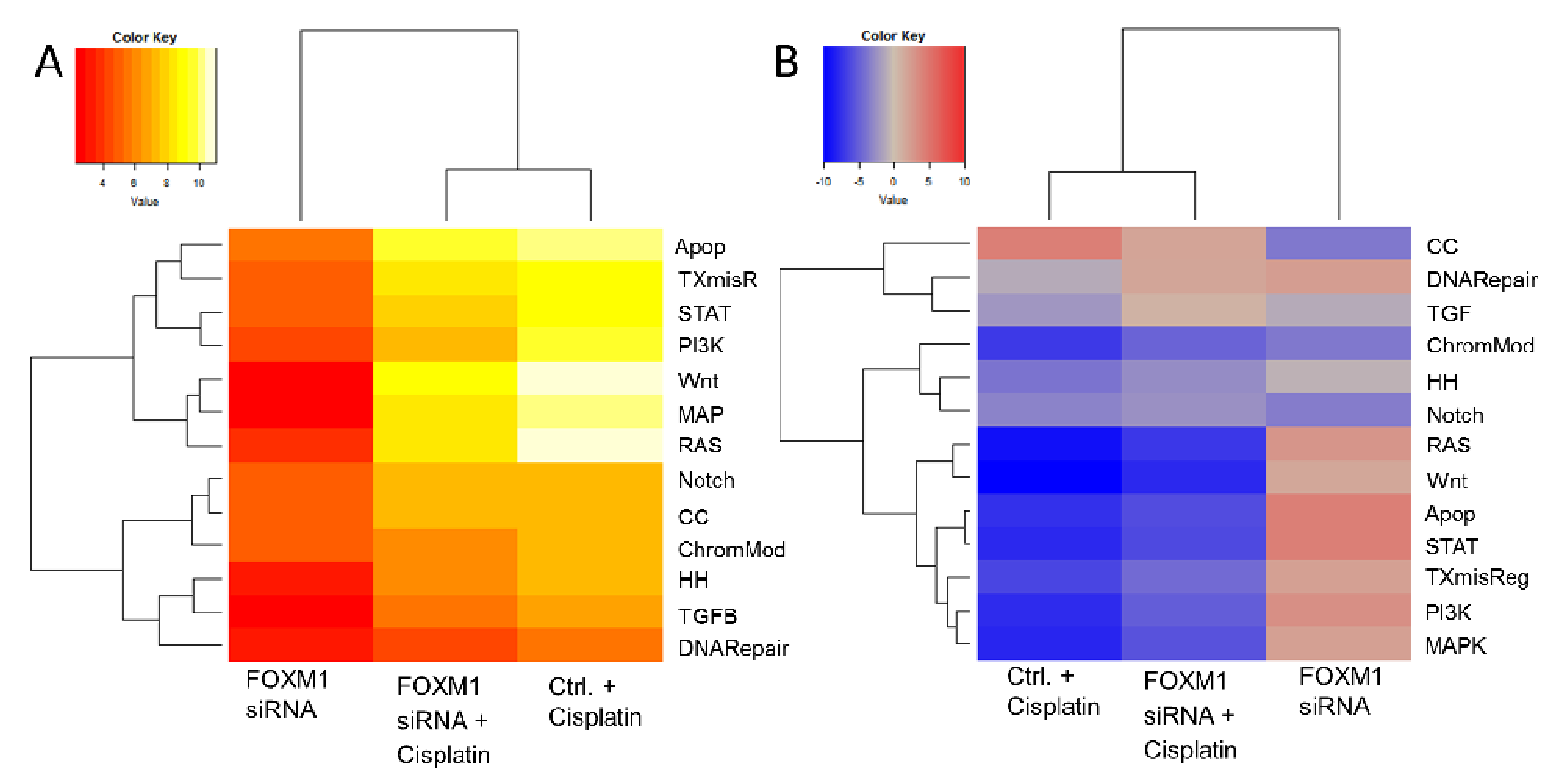

Figure 4: Gene expression analysis of $\mathrm{NCl}-\mathrm{H} 69$
after knockdown of FOXM1 and combination
treatment with cisplatin. A Global significance
score indicate if pathway genes are extensively
differentially expressed (yellow vs. red). Especially
after cisplatin treatment most pathways are
overexpressed. B Directed GSA indicates whether
pathway genes are negatively or positively regulated
compared to a covariate. After knockdown of FOXM1
cell cycle associated genes are downregulated,
whereas apoptosis and STAT pathway related genes
are upregulated. Cisplatin treatment leads to a strong
downregulation of almost all pathways, except for
cell cycle associated genes.

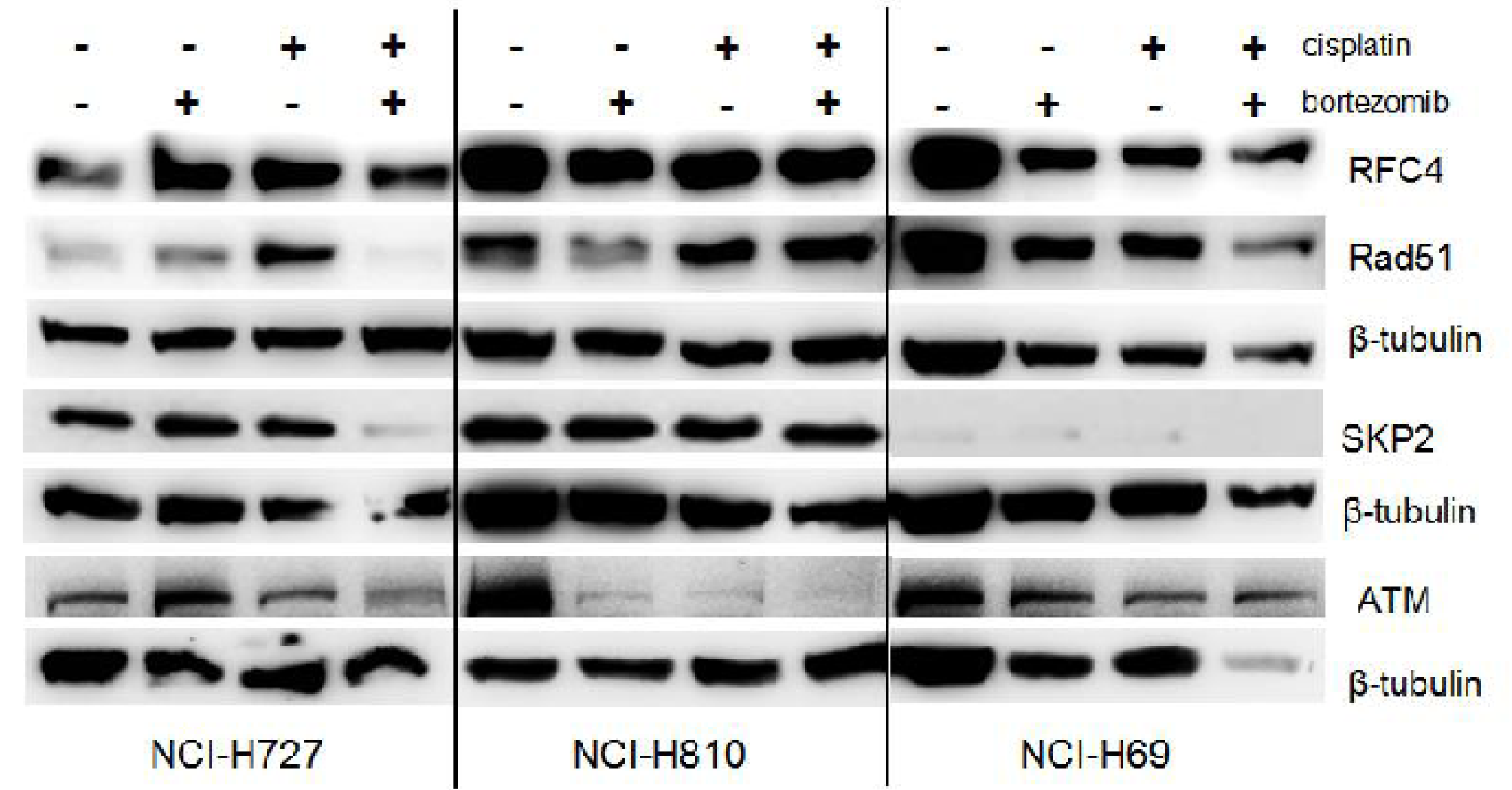

Figure 5: Western Blot analysis of DNA repair associated gene products in BP-NEN after treatmen with bortezomib and cisplatin. After cisplatin treatmen RFC4 and Rad51 are slightly enhanced in all cell lines. Bortezomib treatment as well as combination therapy suppresses protein expression of RFC4, Rad51, and SKP2 in $\mathrm{NCl}-\mathrm{H} 727$ Same tendencies can be seen in the highgrade BP-NEN cell lines. 